\title{
Dix années des Annales françaises de médecine d'urgence
}

\author{
Ten Years of the French Annals of Emergency Medicine
}

\author{
B. Riou $\cdot$ K. Tazarourte \\ pour les membres passés et présents du comité de rédaction des Annales françaises de médecine d'urgence*
}

(C) SFMU et Lavoisier SAS 2021

En 2010, lors du Congrès Urgence de la Société française de médecine d'urgence (SFMU) était publié le numéro 0 des Annales françaises de médecine d'urgence (AFMU), prélude à son lancement en 2011. Après plus de dix ans de fonctionnement, il est utile de faire un point sur notre revue scientifique et ses réalisations. Ce bilan complète l'avis de nos lecteurs récemment interrogés [1].

L'objectif de la SFMU était de faire de cette revue une des toutes premières revues francophones de médecine d'urgence. Le contrat semble rempli si l'on considère le nombre de personnes qui reçoivent les AFMU, près de 2000 cette année, la régularité de parution tout comme la diversité et la qualité des articles publiés. Le Syndicat de la presse et de l'édition des professions de santé (SPEPS) a décerné à deux reprises un prix aux AFMU, dans la catégorie « travaux originaux » [2], puis dans la catégorie « éditorial » [3]. Bien qu'étant la revue d'expression scientifique d'une société savante française, les AFMU se sont d'emblée positionnées sur le terrain de la francophonie en accueillant en permanence, au sein de leur comité de rédaction, des représentants de la Belgique et de la Suisse, et ont publié des travaux d'équipes francophones.

Le tableau 1 résume les publications des AFMU au cours de ces dix dernières années, totalisant plus de 1000 manuscrits. En dehors des articles de commande (éditoriaux, actualités), tous les articles publiés dans les AFMU sont soumis à un processus d'expertise comportant la relecture par deux experts (Tableau 2). La sélection est exigeante comme

\footnotetext{
B. Riou $(\square)$

Rédacteur en chef des Annales françaises de médecine d'urgence, service des urgences, CHU Pitié-Salpêtrière, Sorbonne

Université, Assistance publique-Hôpitaux de Paris (AP-HP),

F-75013 Paris, France

e-mail : bruno.riou@aphp.fr

\section{K. Tazarourte}

Président de la Société française de médecine d'urgence,

Samu-Smur 69 et service des urgences, hôpital Édouard-Herriot, hospices civils de Lyon, université Claude-Bernard-Lyon-I, F-69437 Lyon cedex 03, France
}

en témoigne un taux d'acceptation de $48 \%$ pour les articles originaux, de $45 \%$ pour les cas cliniques et de $57 \%$ pour les images en médecine d'urgence. Le taux élevé d'acceptation des mises au point (97\%) s'explique par le fait qu'il s'agit le plus souvent d'une sollicitation des AFMU auprès d'auteurs ayant démontré une maîtrise préalable du domaine et que les révisions successives permettent le plus souvent d'améliorer les manuscrits jusqu'à leur acceptation, ce qui n'est pas toujours possible avec les autres types de manuscrits. La liste des relecteurs est publiée à l'issue de chaque année, la plupart d'entre eux ayant une expérience conséquente des publications au niveau international [4]. La durée du processus éditorial est satisfaisante comme le montrent des délais médians de première réponse de 18 jours pour les articles originaux et de 29 jours pour les mises au point, et un délai médian de publication des articles acceptés de 55 jours pour les articles originaux et de 69 jours pour les mises au point. Les AFMU ont ainsi rempli leur objectif de réponse rapide donnée aux manuscrits soumis et de publication rapide des manuscrits acceptés, en particulier avec la mise en ligne précédant la publication sous format papier.

Pendant cette décennie, la médecine d'urgence a dû faire face à des crises en rapport avec les attentats terroristes ou plus récemment avec la pandémie de Covid. Les AFMU ont réagi avec promptitude en permettant la publication d'un retour d'expérience des attentats de novembre 2015 deux mois plus tard [5] et de la crise Covid dès septembre 2020, entre les première et deuxième vagues épidémiques [6].
* Membres passés et présents du comité de rédaction des AFMU : Frédéric Adnet (Bobigny), Françoise Carpentier (Grenoble), PierreNicolas Carron (Lausanne), Hélène Chappuy (Paris), Sandrine Charpentier (Toulouse), Yann-Erick Claessens (Monaco), Gérard Chéron (Paris), Pierre-Géraut Claret (Nîmes), Xavier Combes (Bordeaux), Guillaume Debaty (Grenoble), Thibaut Desmettre (Besançon), Florence Dumas (Paris), Agnès Hibon-Ricard (Pontoise), Patricia Jabre (Paris), Luc-Marie Joly (Rouen), Yonathan Freund (Paris), Frédéric Lapostolle (Bobigny), Dominique Lauque (Toulouse), Jacques Levraut (Nice), Emmanuel Montassier (Nantes), Andrea Penaloza (Bruxelles), Patrick Ray (Dijon), Bertrand Renaud (Paris), Frédéric Thys (Bruxelles), Bertrand Yersin (Lausanne) 
Tableau 1 Nombre d'articles publiés dans les Annales françaises de médecine d'urgence depuis dix ans

\begin{tabular}{|llllllllllll}
\hline Rubrique & $\mathbf{2 0 1 1}$ & $\mathbf{2 0 1 2}$ & $\mathbf{2 0 1 3}$ & $\mathbf{2 0 1 4}$ & $\mathbf{2 0 1 5}$ & $\mathbf{2 0 1 6}$ & $\mathbf{2 0 1 7}$ & $\mathbf{2 0 1 8}$ & $\mathbf{2 0 1 9}$ & $\mathbf{2 0 2 0}$ & Total \\
\hline Éditoriaux & 12 & 9 & 6 & 8 & 10 & 6 & 6 & 8 & 7 & 4 & 76 \\
Articles originaux & 13 & 15 & 19 & 17 & 24 & 27 & 23 & 24 & 24 & 24 & 210 \\
Mises au point & 15 & 20 & 21 & 14 & 11 & 10 & 10 & 10 & 7 & 7 & 125 \\
Cas cliniques & 27 & 24 & 33 & 31 & 22 & 21 & 17 & 22 & 22 & 13 & 232 \\
Images en médecine d'urgence & 22 & 27 & 55 & 30 & 34 & 17 & 19 & 17 & 12 & 7 & 240 \\
Recommandations & 3 & 3 & 0 & 2 & 1 & 2 & 2 & 3 & 2 & 2 & 20 \\
Lettres à la rédaction & 2 & 0 & 0 & 1 & 0 & 4 & 0 & 0 & 3 & 0 & 10 \\
Actualités en médecine d'urgence & 6 & 5 & 6 & 6 & 6 & 6 & 6 & 6 & 6 & 6 & 59 \\
Pearls Cochrane & - & - & - & - & - & - & - & 4 & 6 & 5 & 15 \\
Note de lecture & 2 & 4 & 2 & 2 & 0 & 0 & 2 & 2 & 2 & 3 & 19 \\
Total & 102 & 107 & 142 & 111 & 108 & 93 & 85 & 96 & 91 & 71 & 1006
\end{tabular}

Les articles de retour d'expérience ont été inclus dans les articles originaux. Les articles des rubriques « Gestes en médecine d'urgence » et « méthodologie » ont été inclus dans les mises au point. Les « cas cliniques commentés » ont été inclus dans les « cas cliniques »

\begin{tabular}{|c|c|c|c|c|c|c|c|}
\hline Catégories & $\begin{array}{l}\text { Nombre } \\
\text { de manuscrits } \\
\text { soumis }\end{array}$ & $\begin{array}{l}\text { Proportion } \\
\text { de manuscrits } \\
\text { acceptés (\%) }\end{array}$ & $\begin{array}{l}\text { Nombre moyen } \\
\text { de relecteurs } \\
\text { par manuscrit }\end{array}$ & $\begin{array}{l}\text { Acceptation } \\
\text { après une } \\
\text { révision }(\%)\end{array}$ & $\begin{array}{l}\text { Acceptation } \\
\text { après deux } \\
\text { révisions } \\
\text { ou plus }(\%)\end{array}$ & $\begin{array}{l}\text { Délai } \\
\text { de la première } \\
\text { réponse }(\mathbf{j})\end{array}$ & $\begin{array}{l}\text { Délai } \\
\text { pour la réponse } \\
\text { finale } \\
\text { des manuscrits } \\
\text { acceptés }(j)\end{array}$ \\
\hline $\begin{array}{l}\text { Article } \\
\text { original }\end{array}$ & 1039 & 48 & 2,3 & 46 & 54 & 18 [16-18] & 55 [41-89] \\
\hline $\begin{array}{l}\text { Mise au } \\
\text { point }\end{array}$ & 345 & 97 & 2,2 & 61 & 49 & 29 [25-29] & 61 [37-69] \\
\hline $\begin{array}{l}\text { Cas } \\
\text { clinique }\end{array}$ & 1266 & 45 & 2,2 & 46 & 54 & $11[12-21]$ & 92 [79-102] \\
\hline Image & 890 & 57 & 1,9 & 59 & 41 & 23 [23-25] & $54[48-72]$ \\
\hline
\end{tabular}

À chaque fois, ces numéros spéciaux ont été publiés en accès libre afin de faciliter la diffusion de l'information.

Les AFMU s'inscrivent dans les règles et les exigences internationales des revues scientifiques. Ainsi, les instructions aux auteurs soulignent la nécessité de respecter les dispositions éthiques, légales et réglementaires applicables à la recherche sur des sujets humains, les règles concernant le rôle et la responsabilité des auteurs, et les principes de l'intégrité scientifique. Les AFMU exhortent les auteurs à être vigilants pour ne pas citer en référence des revues prédatrices [7] et ont relayé la liste des revues de médecine d'urgence recommandées par le CNU de médecine d'urgence [8]. Au cours de cette décennie, deux tentatives de plagiat ont été détectées et ont fait l'objet d'éviction des auteurs de toute publication pendant plusieurs années et d'alertes auprès des autorités de tutelle des auteurs fautifs [9]. De nombreuses maladresses dans la citation appropriée des sources ont été corrigées pendant le processus éditorial.

Pour conclure ce bref bilan de dix années de fonctionnement, nous souhaitons remercier chaleureusement nos auteurs et nos relecteurs qui ont permis que cette revue scientifique vive et participe à la dissémination des connaissances nouvelles en médecine d'urgence. Nos remerciements vont également aux membres du comité de rédaction, qui se sont relayés au cours de ces années et aux éditeurs, Springer puis Lavoisier, qui nous ont accompagnés dans cette aventure pour œuvrer à la réalisation au quotidien de ce qui correspond à un cumul de près de 4500 pages. Les AFMU étant 
solidement implantées dans le paysage de la médecine d'urgence, il convient de poursuivre nos efforts dans le futur pour les faire grandir encore et répondre aux attentes de nos lecteurs [1].

Liens d'intérêts : les auteurs déclarent ne pas avoir de liens d'intérêts.

\section{Références}

1. Claret PG, Riou B (2021) L'avis de nos lecteurs. Ann Fr Med Urgence 11:73-5

2. Claudet I, Grouteau E (2011) Préparation des services d'accueil des urgences (SAU) français à la prise en charge des urgences vitales de l'enfant. Ann Fr Med Urgence 1:5-9
3. Riou B (2013) Les urgences : une solution et non un problème ! Ann Fr Med Urgence 3:1-2

4. Annales françaises de médecine d'urgence (2021) Remerciements. Ann Fr Med Urgence 11:71-2

5. Riou B (2016) 13 novembre 2015 : terrorisme, résilience et espoir. Ann Fr Med Urgence 6:1-2

6. Riou B (2021) Retour d'expérience de la crise Covid-19 en médecine d'urgence. Ann Fr Med Urgence 10:197-8

7. De La Blanchardière A, Barde F, Peiffer-Smadja N, Maisonneuve H (2021) Revues prédatrices : une vraie menace pour la recherche médicale. 1. Identifier ces revues et comprendre leur fonctionnement. Rev Med Interne 42:(in press)

8. Riou B, Carli P, Charpentier S, et al (2018) Modalités de fonctionnement du Conseil national des universités (CNU) de médecine d'urgence. Ann Fr Med Urgence 8:1-6

9. Bergadaa M (2015) Le plagiat académique. Comprendre pour agir. L'Harmattan, Paris 